\title{
SPURIOUS REFLECTION OF ELASTIC WAVES DUE TO GRADUALLY CHANGING FINITE ELEMENT SIZE
}

\author{
ZEKAI CELEP† AND ZDENĚK P. BAŽANT \\ Centre for Concrete and Geomaterials, The Technological Institute, Northwestern University, Evanston, Illinois, U.S.A.
}

SUMMARY

When a homogeneous elastic continuum is modelled by a non-uniform finite element grid, the differences in the size of adjacent finite elements can cause a spurious wave reflection which does not exist in the continuum. Extending previous studies in which the spurious wave reflection was investigated for the case of two uniform grids with a sudden element size change from one grid to the other, the present study deals with the case when the two uniform grids of different element sizes are separated by a transition zone through which the element size varies gradually, either by a geometric progression or by an arithmetic progression. The solution is carried out in complex variables and numerical plots of the results are given. When the ratio of wavelength to the largest element size is as low as $4: 1$, the spurious wave reflection is very significant, while for the ratios over $10: 1$ it is insignificant. Inserting a transition zone with gradually varying element size between the two uniform parts of the grid somewhat mitigates the phenomenon of spurious reflection, but this is significant only when the ratio of element sizes in the uniform grids is small (less than about $1 \cdot 5: 1$ ). Varying the element size throughout the transition zone in an arithmetic progression seems slightly better than in a geometric progression. The spurious wave reflection is less pronounced for higher-order elements, in particular for linear strain elements as compared to constant strain elements. The spurious reflection is also less severe for consistent mass than for lumped mass.

\section{INTRODUCTION}

When an elastic wave passes through a finite element grid and the wavelength is not much larger than the largest element size, spurious wave reflections are caused by changes in the finite element size. Expanding previous studies of wave dispersion due to various types of discretization, ${ }^{1,2}$ Holmes and Belytschko ${ }^{3}$ and others ${ }^{4-8}$ numerically demonstrated the noise due to changes in element size. In two preceding studies, ${ }^{9.10}$ generalizing the classical analysis of Brillouin ${ }^{11}$ devoted to wave propagation in microscopic crystal lattices, the amplitudes and energy fluxes of the spurious reflected waves were solved analytically for the case of two half-planes each of which is subdivided by a uniform grid while the element size changes suddenly across the interface of the half-planes. Both for constant strain elements ${ }^{9}$ and for elements with linear strain distribution, ${ }^{10}$ it was found that the sudden change in the element size can cause a significant fraction of the energy flux to get reflected from the boundary between finite elements of different sizes.

It will be the purpose of this investigation to study the spurious reflection when the element size is changed gradually over a transition zone of several elements. We will confine our attention to a homogeneous medium, in which no wave reflections occur. Thus, the reflected

$\uparrow$ Post-Doctoral Research Associate, Associate Professor on leave from Technical University, Istanbul. $¥$ Professor of Civil Engineering and Director. 
waves in the finite element grid are spurious in nature. We will solve the problem both for constant strain finite elements and for finite elements with a linear strain distribution.

We consider a longitudinal elastic wave with a planar wavefront arriving from the left. We assume the grid in the homogeneous medium to be chosen as rectangular, the nodal lines being parallel to the direction of the incident wave. In such a situation we have a translation symmetry parallel to the wavefront, and therefore it suffices to solve a one-dimensional problem with finite elements on one line (Figure 1). The constant-strain finite elements have two nodes, and the linear-strain finite elements have three nodes one of which is an interior node.

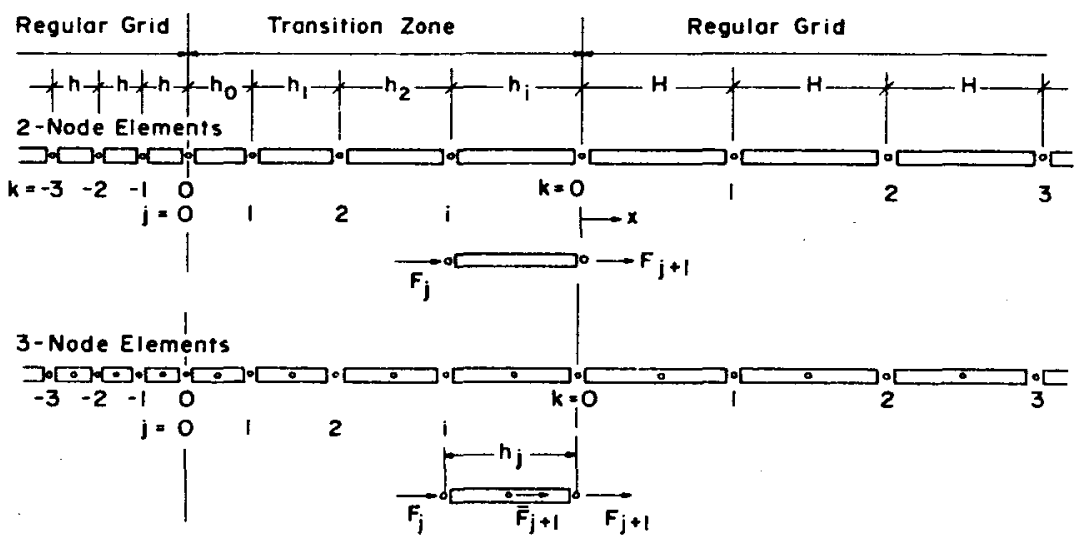

Figure 1. Element arrangement, numbering and notation

\section{CONSTANT-STRAIN FINITE ELEMENTS}

Let $h_{i}$ denote the length of the $j$ th finite element, and let its thickness be 1 . The mass of the continuum within the element length is $\rho h_{j}$, where $\rho=$ mass density of the continuum. We consider that mass $m \rho h_{j}$ is uniformly distributed over the element and the remaining mass $(1-m) \rho h_{j}$ is lumped into the two nodes. Thus the mass of the node is $(1-m)\left(h_{j-1}+h_{j}\right) / 2$. The nodal forces acting on the $j$ th element may now be expressed as

$$
\left\{\begin{array}{c}
F_{j} \\
F_{j+1}
\end{array}\right\}=\frac{E^{\prime}}{h_{j}}\left[\begin{array}{rr}
1 & -1 \\
-1 & 1
\end{array}\right]\left\{\begin{array}{c}
u_{j} \\
u_{j+1}
\end{array}\right\}+\frac{m \rho h_{j}}{6}\left[\begin{array}{ll}
2 & 1 \\
1 & 2
\end{array}\right]\left\{\begin{array}{c}
\ddot{u}_{j} \\
\ddot{u}_{j+1}
\end{array}\right\}
$$

where $E^{\prime}=$ Young's elastic modulus. The equation of motion of node $j$ may then be written as

$$
\frac{m}{6} \rho\left[h_{j-1}\left(\ddot{u}_{j-1}+2 \ddot{u}_{j}\right)+h_{j}\left(2 \ddot{u}_{j}+\ddot{u}_{j+1}\right)\right]+\frac{1-m}{2}\left(h_{j-1}+h_{j}\right) \ddot{u}_{i}=\frac{E^{\prime}}{h_{j-1}}\left(u_{j-1}-u_{j}\right)-\frac{E^{\prime}}{h_{j}}\left(u_{j}-u_{j+1}\right)
$$

For the purpose of numerical analysis we consider the second time derivatives, denoted by superimposed double dots, to be replaced by the finite difference expression

$$
\ddot{u}_{i} \simeq \frac{1}{\tau^{2}}\left(u_{j, r+1}-2 u_{j, r}+u_{j, r-1}\right)
$$

in which $\tau=$ the time step and $r=$ the number of the time step $(r=1,2, \ldots)$.

We consider that for the node numbers $k \leqslant 0(k=\ldots-3,-2,-1)$ the size of the finite element is uniform and within this region we seek the solution in the form

$$
u_{k}(x, t)=\mathrm{e}^{i \omega(x-v t)}+A \mathrm{e}^{i \omega(-x-v t)}=\mathrm{e}^{i \omega(k h-v r r)}+A \mathrm{e}^{i \omega(-k h-v r \tau)}
$$


in which the first term represents the incident (arriving) wave and the second term the spurious reflected wave (if any). Substituting equation (3) into equation (2) with equation (2a), we obtain

$$
\frac{v^{2}}{v_{0}^{2}}\left[1-m+\frac{m}{3}(2+\cos \omega h)\right]=\frac{1}{S} \frac{\sin ^{2}(\omega h / 2)}{\left(\omega^{2} h^{2} / 4\right)}
$$

where

$$
S=\sin ^{2}(\omega v \tau / 2) /\left(\omega^{2} v^{2} \tau^{2} / 4\right) \quad(S \rightarrow 1, \text { for } \tau \rightarrow 0)
$$

and

$$
v_{0}^{2}=E^{\prime} / \rho \quad\left(v \rightarrow v_{0} \text { for } \tau \rightarrow 0, h \rightarrow 0\right)
$$

We further assume that the elements on the right-hand side of the grid, numbered as $K=1,2,3, \ldots$, are also uniform although with a different size, $H$. We will seek the refracted (diffracted) wave passing to the right side of the grid in the form

$$
u_{k}=D \mathrm{e}^{i \Omega(x-V t)}=D \mathrm{e}^{i \Omega(K H-V t)}
$$

For the refracted wave velocity we obtain an equation similar to equation (4):

$$
\frac{V^{2}}{v_{0}^{2}}\left[1-m+\frac{m}{3}(2+\cos \Omega H)\right]=\frac{1}{\bar{S}} \frac{\sin ^{2}(\Omega H / 2)}{\left(\Omega^{2} H^{2} / 4\right)}
$$

in which

$$
\bar{S}=\sin ^{2}(\Omega V \tau / 2) /\left(\Omega^{2} V^{2} \tau^{2} / 4\right)
$$

Between the two uniform parts of the grid extending to the infinity at left and right we consider a finite number of transition elements the size of which gradually varies. We number them as $j=1,2, \ldots, i$. We seek the solution for these elements in the form

$$
u_{j}=A_{J} \mathrm{e}^{-i \omega_{,} v_{j} t}=A_{j} \mathrm{e}^{-i \omega_{i} v_{j} r \tau} \quad(j=1,2, \ldots, i)
$$

Here the amplitudes at the transition nodes, $A_{i}$, are complex numbers. The number of transition nodes is $i$ and the number of transition elements is $i+1$. Writing the equation of motion for the nodes $k=0, j=1,2, \ldots, i$, and $K=0$, we conclude that

$$
\omega v=\Omega V=\omega_{j} v_{i} \quad(\text { for } j=1,2, \ldots, i)
$$

For $k=0$ we further obtain

$$
\begin{aligned}
A & \left.\frac{S \omega^{2} h^{2} v^{2}}{v_{0}^{2}}\left[\frac{m}{3}\left(2+p+2 \frac{h_{0}}{h}\right)+(1-m)\left(1+\frac{h_{0}}{h}\right)\right]-2\left(1+\frac{h}{h_{0}}\right)+2 p\right\} \\
& +A_{1}\left[\frac{S \omega^{2} h^{2} v^{2}}{3 v_{0}^{2}} \frac{h_{0}}{h}+2 \frac{h}{h_{0}}\right]=-\frac{S \omega^{2} h^{2} v^{2}}{v_{0}^{2}}\left\{\frac{m}{3}\left(2+p^{-1}+\frac{2}{3} \frac{h_{0}}{h}\right)\right. \\
& \left.+(1-m)\left(1+\frac{h_{0}}{h}\right)-2\left(1+\frac{h}{h_{0}}\right)+2 p^{-1}\right\}
\end{aligned}
$$

for $j=1$ :

$$
\begin{aligned}
A\left[\frac{S \omega^{2} h^{2} v^{2}}{v_{0}^{2}} \frac{m}{3}+2 \frac{h^{2}}{h_{0}^{2}}\right] & +A_{1}\left[\frac{S \omega^{2} h^{2} v^{2}}{v_{0}^{2}}\left(1+\frac{h_{1}}{h_{0}}\right)\left(1-\frac{m}{3}\right)-2 \frac{h^{2}}{h_{0}^{2}}\left(1+\frac{h_{0}}{h_{1}}\right)\right] \\
& +A_{2}\left[\frac{S \omega^{2} h^{2} v^{2}}{3 v_{0}^{2}} \frac{m h_{1}}{h_{0}}+2 \frac{h^{2}}{h_{0} h_{1}}\right]=-\frac{S \omega^{2} h^{2} v^{2} m}{3 v_{0}^{2}}-2 \frac{h^{2}}{h_{0}^{2}}
\end{aligned}
$$


for $j=2,3,4, \ldots, i-1$ :

for $j=i$ :

$$
\begin{aligned}
& A_{j-1}\left[\frac{S \omega^{2} h^{2} v^{2}}{v_{0}^{2}} \frac{m}{3}+2 \frac{h^{2}}{h_{i-1}^{2}}\right]+A_{j}\left[\frac{S^{2} \omega^{2} h^{2} v^{2}}{v_{0}^{2}}\left(1+\frac{h_{j}}{h_{j-1}}\right)\left(1-\frac{m}{3}\right)-2 \frac{h^{2}}{h_{j-1}^{2}}\left(1+\frac{h_{j-1}}{h_{j}}\right)\right] \\
& +A_{j+1}\left[\frac{S \omega^{2} h^{2} v^{2}}{v_{0}^{2}} \frac{m}{3} \frac{h_{j}}{h_{j-1}}+2 \frac{h^{2}}{h_{j-1} h_{j}}\right]=0
\end{aligned}
$$

$$
\begin{aligned}
A_{i-1} & {\left[\frac{S \omega^{2} h^{2} v^{2}}{v_{0}^{2}} \frac{m}{3}+2 \frac{h^{2}}{h_{i-1}^{2}}\right]+A_{i}\left[\frac{S \omega^{2} h^{2} v^{2}}{v_{0}^{2}}\left(1+\frac{h_{i}}{h_{i-1}}\right)\left(1-\frac{m}{3}\right)-2 \frac{h^{2}}{h_{i-1}^{2}}\left(1+\frac{h_{i-1}}{h_{i}}\right)\right] } \\
& +D\left[\frac{S \omega^{2} h^{2} v^{2}}{v_{0}^{2}} \frac{m}{3} \frac{h_{i}}{h_{i-1}}+2 \frac{h^{2}}{h_{i-1} h_{i}}\right]=0
\end{aligned}
$$

and finally for $K=0$ :

$$
\begin{aligned}
A_{i}[ & \left.\frac{S \omega^{2} h^{2} v^{2}}{v_{0}^{2}} \frac{m}{3}+2 \frac{h^{2}}{h_{i}^{2}}\right]+D \frac{S \omega^{2} h^{2} v^{2}}{v_{0}^{2}}\left[\frac{m}{3}\left(2+\frac{H}{h_{i}} P+2 \frac{H}{h_{i}}\right)+(1-m)\left(1+\frac{H}{h_{i}}\right)\right] \\
& -\frac{2 h^{2}}{h_{i}^{2}}\left(1+\frac{h_{i}}{H}\right)+2 \frac{h^{2}}{h_{i} H} P=0
\end{aligned}
$$

where $p=\mathrm{e}^{i \omega h}$ and $P=\mathrm{e}^{i \Omega H}$. Using equations (4), (6) and (8), we obtain for the transmitted wave the relation

$$
\cos \Omega H=\frac{\left(\frac{H^{2}}{h^{2}}-1\right)[m(1-\cos \omega h)-3]+3 \frac{H^{2}}{h^{2}} \cos \omega h}{m\left(\frac{H^{2}}{h^{2}}-1\right)(1-\cos \omega h)+3}
$$

The energy flux to the right is given by the expression $\mathscr{P}=\operatorname{Re}\left(F_{j}\right) \operatorname{Re}\left(u_{j}\right)$, which yields

$$
\mathscr{P}=\left[\frac{E}{h_{j-1}} \operatorname{Re}\left(u_{j}-u_{j-1}\right)+\frac{m \rho h_{j-1}}{6} \operatorname{Re}\left(2 \ddot{u}_{j}+\ddot{u}_{j-1}\right)\right] \operatorname{Re}\left(u_{j}\right)
$$

Denoting the energy fluxes of the incident, reflected and refracted wave as $\mathscr{P}_{1}, \mathscr{P}_{A}, \mathscr{P}_{D}$, we obtain for the time-average energy fluxes in the uniform parts of the finite element grid the following expressions:

$$
\begin{array}{ll}
\left\langle\mathscr{P}_{1}\right\rangle=-\frac{\pi E^{\prime}}{h}\left(1+\frac{m \omega^{2} h^{2}}{6} \frac{v^{2}}{v_{0}^{2}}\right) \sin \omega h & \text { (incident } \rightarrow) \\
\left\langle\mathscr{P}_{A}\right\rangle=\frac{\pi E^{\prime}}{h}\left(1+\frac{m \omega^{2} h^{2}}{6} \frac{v^{2}}{v_{0}^{2}}\right)\left(a_{r}^{2}+a_{i}^{2}\right) \sin \omega h & (\text { reflected } \leftarrow) \\
\left\langle\mathscr{P}_{D}\right\rangle=-\frac{\pi E^{\prime}}{H}\left(1+\frac{m \omega^{2} H^{2}}{6} \frac{V^{2}}{v_{0}^{2}}\right)\left(d_{r}^{2}+d_{i}^{2}\right) \sin \Omega H & (\text { refracted } \rightarrow)
\end{array}
$$

in which $A=a_{r}+i a_{i}$ and $D=d_{r}+i d_{i}$. Consideration of energy conservation requires that

$$
\left\langle\mathscr{P}_{1}\right\rangle=\left\langle\mathscr{P}_{A}\right\rangle+\left\langle\mathscr{P}_{D}\right\rangle
$$

This relation serves as a check of the numerical calculations. 


\section{FINITE ELEMENTS WITH LINEAR STRAIN DISTRIBUTION}

Denoting again as $m$ the portion of mass which is uniformly distributed throughout the finite element, the additional lumped mass in the centre node of the $j$ th element is $\rho(1-m) h_{j} / 2$ and the additional lumped mass in the node between elements $j-1$ and $j$ is $\rho(1-m) \times$ $\left(h_{j-1}+h_{j}\right) / 4$ (Figure 1$)$. The nodal forces acting on the $j$ th 3 -node element may now be expressed as

$$
\left\{\begin{array}{c}
F_{j} \\
\bar{F}_{j} \\
F_{j+1}
\end{array}\right\}=\frac{E^{\prime}}{3 h_{j}}\left[\begin{array}{rrr}
7 & -8 & 1 \\
-8 & 16 & -8 \\
1 & -8 & 7
\end{array}\right]\left\{\begin{array}{c}
u_{j} \\
v_{j} \\
u_{j+1}
\end{array}\right\}+\frac{m \rho h_{j}}{30}\left[\begin{array}{rrr}
4 & 2 & -1 \\
2 & 16 & 2 \\
-1 & 2 & 4
\end{array}\right]\left\{\begin{array}{c}
\ddot{u}_{i} \\
\ddot{v}_{i} \\
\ddot{u}_{j+1}
\end{array}\right\}
$$

The equation of motion for the interelement node $j$ is

$$
\begin{gathered}
(1-m) \frac{\rho\left(h_{j-1}+h_{j}\right)}{4} \ddot{u}_{j}+\frac{m \rho}{30}\left[h_{j-1}\left(-\ddot{u}_{j-1}+2 \ddot{v}_{j-1}+4 \ddot{u}_{j}\right)+h_{j}\left(4 \ddot{u}_{j}+2 \ddot{v}_{i}-\ddot{u}_{j+1}\right)\right] \\
=\frac{E^{\prime}}{3 h_{j-1}}\left(-u_{j-1}+8 v_{j-1}-7 u_{j}\right)-\frac{E^{\prime}}{3 h_{j}}\left(7 u_{j}-8 v_{j}+u_{j+1}\right)
\end{gathered}
$$

and the equation of motion for the interior node of element $j$ is

$$
(1-m) \frac{\rho h_{j}}{2} \ddot{v}_{j}+\frac{m \rho h_{j}}{15}\left(\ddot{u}_{j}+8 \ddot{v}_{i}+\ddot{u}_{j+1}\right)=\frac{8 E^{\prime}}{3 h_{j}}\left(u_{j}-2 v_{j}+u_{j+1}\right)
$$

We seek the incident and reflected waves within the left uniform part of the grid $(k=\ldots$, $-3,-2,-1)$ in the form

$$
\begin{aligned}
& u_{k}=\mathrm{e}^{i \omega(k h-v r r)}+A \mathrm{e}^{i \omega(-k h-v r r)} \\
& v_{k}=B \mathrm{e}^{i \omega[(k+1 / 2) h-v r r]}+C \mathrm{e}^{i \omega[-(k+1 / 2) h-v r r]}
\end{aligned}
$$

where $u_{k}$ is the displacement of the interelement node and $v_{k}$ is the displacement of the interior node of the element. Substituting equation (19) into equation (2) with equation (2a), we obtain

in which

$$
a u_{k}=b\left(v_{k-1}+v_{k}\right), \quad c v_{k}=b\left(u_{k}+u_{k+1}\right)
$$

$$
\begin{aligned}
& a=\frac{2}{3}(\cos \omega h+7)-S \frac{\omega^{2} h^{2} v^{2}}{v_{0}^{2}}\left[\frac{m}{15}(4-\cos \omega h)+\frac{1-m}{2}\right] \\
& b=\frac{8}{3}+S \frac{\omega^{2} h^{2} v^{2}}{v_{0}^{2}} \frac{m}{15} \\
& c=\frac{16}{3}-S \frac{\omega^{2} h^{2} v^{2}}{v_{0}^{2}}\left(\frac{8 m}{15}+\frac{1-m}{2}\right), \quad S=\sin ^{2} \frac{\omega v \tau}{2} / \frac{\omega^{2} v^{2} \tau^{2}}{4}
\end{aligned}
$$

The two relations in equation (20) yield the following relation for the wave velocity $b$ :

$$
a c=2 b^{2}(1+\cos \omega h)
$$

This can be also expressed as

$$
c_{1}\left(\frac{v}{v_{0}}\right)^{4}+c_{2}\left(\frac{v}{v_{0}}\right)^{2}+c_{3}=0
$$


in which

$$
\begin{aligned}
& c_{1}=S^{2} \omega^{4} h^{4}\left[\frac{2 m^{2}}{45}(\cos \omega h-3)+\frac{m(1-m)}{30}(\cos \omega h-12)-\frac{(1-m)^{2}}{4}\right] \\
& c_{2}=\frac{2}{3} S \omega^{2} H^{2}\left[\frac{104}{15} m+\left(\frac{16 m}{15}+\frac{1-m}{2}\right) \cos \omega h+\frac{15}{2}(1-m)\right] \\
& c_{3}=\frac{32}{3}(\cos \omega h-1)
\end{aligned}
$$

The same procedure can be carried out for the right uniform part of the grid $(K=1,2,3, \ldots)$. We seek the solution for the refracted wave in the form

$$
u_{K}=D \mathrm{e}^{i \Omega(K H-V r r)}, \quad v_{K}=E \mathrm{e}^{i \Omega[(K+1 / 2) H-V r r]}
$$

in which

$$
\bar{a} u_{K}=\bar{b}\left(v_{K-1}+v_{K}\right), \bar{c} v_{K}=\bar{b}\left(u_{K}+u_{K+1}\right),
$$

with the notation

$$
\begin{aligned}
& \bar{a}=\frac{2}{3}(\cos \Omega H+7)-S \frac{\Omega^{2} H^{2} V^{2}}{v_{0}^{2}}\left[\frac{m}{15}(4-\cos \Omega H)+\frac{1-m}{2}\right] \\
& \bar{b}=\frac{8}{3}+S \frac{\Omega^{2} H^{2} V^{2}}{v_{0}^{2}} \frac{m}{15} \\
& \bar{c}=\frac{16}{3}-S \frac{\Omega^{2} H^{2} V^{2}}{v_{0}^{2}}\left(\frac{8 m}{15}+\frac{1-m}{2}\right) \quad S=\sin ^{2} \frac{\Omega V \tau}{2} / \frac{\Omega^{2} V^{2} \tau^{2}}{4}
\end{aligned}
$$

For the velocity of the refracted wave $V$ we get

$$
\bar{a} \bar{c}=2 \vec{b}^{2}(1+\cos \Omega H)
$$

For the displacements at the transition nodes we seek the solution in the form

$$
\begin{aligned}
& u_{j}=A_{i} \mathrm{e}^{-i \omega_{j} v_{j} \tau \tau} \quad j=1,2, \ldots, i \\
& v_{j}=B_{i} \mathrm{e}^{-i \omega_{j} v_{j} r \tau} \quad j=0,1,2, \ldots, i
\end{aligned}
$$

Substituting this into the equations of motion for the element boundary nodes and the interior nodes, we find that

$$
\omega v=\Omega V=\omega_{j} v_{i} \quad(j=0,1,2, \ldots, i)
$$

Furthermore, we obtain for the boundary node $k=0$ :

$$
\begin{gathered}
A\left\{S \frac{\omega^{2} h^{2} v^{2}}{v_{0}^{2}}\left[\frac{m}{30} p-\left(1+\frac{h_{0}}{h}\right)\left(\frac{2 m}{15}+\frac{1-m}{4}\right)\right]+\frac{p}{3}+\frac{7}{3}\left(1+\frac{h}{h_{0}}\right)\right\}+A_{1}\left[S \frac{\omega^{2} h^{2} v^{2}}{v_{0}^{2}} \frac{m}{30} \frac{h_{0}}{h}+\frac{h}{3 h_{0}}\right] \\
-\left[S \frac{\omega^{2} h^{2} v^{2}}{v_{0}^{2}} \frac{m}{15}+\frac{8}{3}\right]\left(B p^{-1 / 2}+C p^{1 / 2}\right)-B_{0}\left[S \frac{\omega^{2} h^{2} v^{2}}{v_{0}^{2}} \frac{m}{15} \frac{h_{0}}{h}+\frac{8 h}{3 h_{0}}\right] \\
=\frac{1-m}{4}\left(1+\frac{h_{0}}{h}\right)-S \frac{\omega^{2} h^{2} v^{2}}{v_{0}^{2}} \frac{m}{30}\left[p^{-1}-4\left(1+\frac{h_{0}}{h}\right)\right]-\frac{1}{3}\left[p^{-1}+7\left(1+\frac{h_{0}}{h}\right)\right]
\end{gathered}
$$


for the interior node $k=0$ :

$$
\begin{gathered}
A\left[S \frac{\omega^{2} h^{2} v^{2}}{v_{0}^{2}} \frac{m}{15}+\frac{8}{3} \frac{h^{2}}{h_{0}^{2}}\right]+B_{0}\left[S \frac{\omega^{2} h^{2} v^{2}}{v_{0}^{2}}\left(\frac{8 m}{15}+\frac{1-m}{2}\right)-\frac{16}{3} \frac{h^{2}}{h_{0}^{2}}\right]+A_{1}\left[S \frac{\omega^{2} h^{2} v^{2}}{v_{0}^{2}} \frac{m}{15}+\frac{8}{3} \frac{h^{2}}{h_{0}^{2}}\right] \\
=-S \frac{\omega^{2} h^{2} v^{2}}{v_{0}^{2}} \frac{m}{15}-\frac{8}{3} \frac{h^{2}}{h_{0}^{2}}
\end{gathered}
$$

for the element boundary node $j=1$ :

$$
\begin{aligned}
-A & \left(S \frac{\omega^{2} h^{2} v^{2}}{v_{0}^{2}} \frac{m}{30}+\frac{h^{2}}{3 h_{0}^{2}}\right)+B_{0}\left(S \frac{\omega^{2} h^{2} v^{2}}{v_{0}^{2}} \frac{m}{15}+\frac{8}{3} \frac{h^{2}}{h_{0}^{2}}\right) \\
& +A_{1}\left[S \frac{\omega^{2} h^{2} v^{2}}{v_{0}^{2}}\left(1+\frac{h_{1}}{h_{0}}\right)\left(\frac{1-m}{4}+\frac{2 m}{15}\right)-\frac{7 h^{2}}{3 h_{0}^{2}}\left(1+\frac{h_{0}}{h_{1}}\right)\right] \\
& +B_{1}\left(S \frac{\omega^{2} h^{2} v^{2}}{v_{0}^{2}} \frac{m}{15} \frac{h_{1}}{h_{0}}+\frac{8 h^{2}}{3 h_{0} h_{1}}\right)-A_{2}\left(S \frac{\omega^{2} h^{2} v^{2}}{v_{0}^{2}} \frac{m}{30} \frac{h_{1}}{h_{0}}+\frac{h^{2}}{3 h_{1} h_{0}}\right)=S \frac{\omega^{2} h^{2} v^{2}}{v_{0}^{2}} \frac{m}{30}+\frac{h^{2}}{3 h_{0}^{2}}
\end{aligned}
$$

for the interior nodes $j=1,2, \ldots, i-1$ :

$$
\begin{gathered}
A_{j}\left(S \frac{\omega^{2} h^{2} v^{2}}{v_{0}^{2}} \frac{m}{15}+\frac{8}{3} \frac{h^{2}}{h_{j}^{2}}\right)+B_{j}\left[S \frac{\omega^{2} h^{2} v^{2}}{v_{0}^{2}}\left(\frac{8 m}{15}+\frac{1-m}{2}\right)-\frac{16}{3} \frac{h^{2}}{h_{j}^{2}}\right] \\
+A_{i+1}\left(S \frac{\omega^{2} h^{2} v^{2}}{v_{0}^{2}} \frac{m}{15}+\frac{8}{3} \frac{h^{2}}{h_{j}^{2}}\right)=0
\end{gathered}
$$

for the element boundary nodes $j=2,3, \ldots, i-1$ :

$$
\begin{aligned}
-A_{j-1} & \left(S \frac{\omega^{2} h^{2} v^{2}}{v_{0}^{2}} \frac{m}{30}+\frac{h^{2}}{3 h_{j-1}^{2}}\right)+B_{j-1}\left(s \frac{\omega^{2} h^{2} v^{2}}{v_{0}^{2}} \frac{m}{15}+\frac{8}{3} \frac{h^{2}}{h_{j-1}^{2}}\right) \\
+ & A_{j}\left[S \frac{\omega^{2} h^{2} v^{2}}{v_{0}^{2}}\left(1+\frac{h_{j}}{h_{j-1}}\right)\left(\frac{1-m}{4}+\frac{2 m}{15}\right)-\frac{7}{3} \frac{h^{2}}{h_{j-1}^{2}}\left(1+\frac{h_{j-1}}{h_{j}}\right)\right] \\
& +B_{j}\left(S \frac{\omega^{2} h^{2} v^{2}}{v_{0}^{2}} \frac{m}{15} \frac{h_{j}}{h_{j-1}}+\frac{8 h^{2}}{3 h_{j-1} h_{j}}\right)-A_{j+1}\left(s \frac{\omega^{2} h^{2} v^{2}}{v_{0}^{2}} \frac{m}{30} \frac{h_{j}}{h_{j-1}}+\frac{h^{2}}{3 h_{j} h_{j-1}}\right)=0
\end{aligned}
$$

for the interior node $j=i$ :

$$
\begin{gathered}
A_{i}\left(s \frac{\omega^{2} h^{2} v^{2}}{v_{0}^{2}} \frac{m}{15}+\frac{8 h^{2}}{3 h_{i}^{2}}\right)+B_{i}\left[S \frac{\omega^{2} h^{2} v^{2}}{v_{0}^{2}}\left(\frac{8 m}{15}+\frac{1-m}{2}\right)-\frac{16 h^{2}}{3 h_{i}^{2}}\right] \\
+D\left(S \frac{\omega^{2} h^{2} v^{2}}{v_{0}^{2}} \frac{m}{15}+\frac{8}{3} \frac{h^{2}}{h_{i}^{2}}\right)=0
\end{gathered}
$$

for the element boundary node $j=i$ :

$$
\begin{aligned}
-A_{i-1}( & \left.S \frac{\omega^{2} h^{2} v^{2}}{v_{0}^{2}} \frac{m}{30}+\frac{h^{2}}{3 h_{i-1}^{2}}\right)+B_{i-1}\left(s \frac{\omega^{2} h^{2} v^{2}}{v_{0}^{2}} \frac{m}{15}+\frac{8 h^{2}}{3 h_{i-1}^{2}}\right) \\
+A_{i} & {\left[S \frac{\omega^{2} h^{2} v^{2}}{v_{0}^{2}}\left(1+\frac{h_{i}}{h_{i-1}}\right)\left(\frac{1-m}{4}+\frac{2 m}{15}\right)-\frac{7 h^{2}}{3 h_{i-1}^{2}}\left(1+\frac{h_{i-1}}{h_{i}}\right)\right] } \\
+ & B_{i}\left(S \frac{\omega^{2} h^{2} v^{2}}{v_{0}^{2}} \frac{m}{15} \frac{h_{i}}{h_{i-1}}+\frac{8 h^{2}}{3 h_{i-1} h}\right)-D\left(s \frac{\omega^{2} h^{2} v^{2}}{v_{0}^{2}} \frac{m}{30} \frac{h_{i}}{h_{i-1}}+\frac{h^{2}}{3 h_{i-1} h_{i}}\right)=0
\end{aligned}
$$


and finally for the element boundary node $K=0$ :

$$
\begin{aligned}
-A_{i}( & \left(S \frac{\omega^{2} h^{2} v^{2}}{v_{0}^{2}} \frac{m}{30}+\frac{H^{2}}{3 h_{i}^{2}}\right)+B_{i}\left(S \frac{\omega^{2} h^{2} v^{2}}{v_{0}^{2}} \frac{m}{15}+\frac{8 h^{2}}{3 h_{i}^{2}}\right) \\
& +D\left[S \frac{\omega^{2} h^{2} v_{0}^{2}}{v_{0}^{2}}\left(1+\frac{H}{h_{i}}\right)\left(\frac{1-m}{4}+\frac{2 m}{15}\right)-\frac{7 h^{2}}{3 h_{i}^{2}}\left(1+\frac{h_{i}}{H}\right)\right. \\
& \left.-P\left(S \frac{\omega^{2} h^{2} v^{2}}{v_{0}^{2}} \frac{m}{30} \frac{H}{h_{i}}+\frac{h^{2}}{3 h_{i} H}\right)\right]+E P^{1 / 2}\left(S \frac{\omega^{2} h^{2} v^{2}}{v_{0}^{2}} \frac{m}{15} \frac{H}{h_{i}}+\frac{8 h^{2}}{3 h_{i} H}\right)=0
\end{aligned}
$$

where $p=\mathrm{e}^{i \omega h}$ and $P=\mathrm{e}^{i \Omega H}$.

Using equations (22), (28) and (30), we obtain for the transmitted wave:

$$
\cos \Omega H=\frac{\bar{h}_{1} \bar{h}_{2}-2 \bar{h}_{3}^{2}}{3 \bar{h}_{3}^{2}-\bar{h}_{1} \bar{h}_{4}}
$$

in which

$$
\begin{aligned}
& \bar{h}_{1}=\frac{16}{3}-S \frac{\omega^{2} h^{2} v^{2}}{v_{0}^{2}} \frac{H^{2}}{h^{2}}\left(\frac{8 m}{15}+\frac{1-m}{2}\right) \quad \bar{h}_{2}=\frac{14}{3}-S \frac{\omega^{2} h^{2} v^{2}}{v_{0}^{2}} \frac{H^{2}}{h^{2}}\left(\frac{4 m}{15}+\frac{1-m}{2}\right) \\
& \bar{h}_{3}=\frac{8}{3}+S \frac{\omega^{2} h^{2} v^{2}}{v_{0}^{2}} \frac{H^{2}}{h^{2}} \frac{m}{15} \quad \bar{h}_{4}=\frac{2}{3}+S \frac{\omega^{2} h^{2} v^{2}}{v_{0}^{2}} \frac{H^{2}}{h^{2}} \frac{m}{15}
\end{aligned}
$$

Substituting equation (20) into equation (19) we obtain

$$
B=2 \frac{b}{c} \cos \frac{\omega h}{2} \quad C=2 A \frac{b}{c} \cos \frac{\omega h}{2}
$$

and similarly for the refracted wave

$$
E=2 D \frac{\bar{b}}{c} \cos \frac{\Omega H}{2}
$$

The energy flux to the right may be calculated as

$$
\mathscr{P}=\left[\frac{E^{\prime}}{h_{j-1}} \operatorname{Re}\left(u_{j-1}-8 v_{j-1}+7 u_{j}\right)+\frac{m \rho h_{j-1}}{30} \operatorname{Re}\left(-\ddot{u}_{j-1}+2 \ddot{v}_{j-1}+4 \ddot{u}_{i}\right)\right] \operatorname{Re}\left(u_{j}\right)
$$

For this we obtain for the incident wave the time-average flux

$$
\left\langle\mathscr{P}_{1}\right\rangle=-\frac{\pi E^{\prime}}{3 h}\left\{8 b_{r} \sin \frac{\omega h}{2}-\sin \omega h+\frac{m \omega^{2} h^{2} v^{2}}{10 v_{0}^{2}}\left(2 b_{r} \sin \frac{\omega h}{2}-\sin \omega h\right)\right\}
$$

for the reflected wave:

$$
\begin{aligned}
\left\langle\mathscr{P}_{\mathrm{A}}\right\rangle= & \frac{\pi E^{\prime}}{3 h}\left\{8\left(a_{r} c_{r}+a_{i} c_{i}\right) \sin \frac{\omega h}{2}+8\left(a_{r} c_{i}-a_{i} c_{r}\right) \cos \frac{\omega h}{2}-\left(a_{r}^{2}+a_{i}^{2}\right) \sin \omega h\right. \\
& \left.+\frac{m \omega^{2} h^{2} v^{2}}{10 v_{0}^{2}}\left[2\left(a_{r} c_{r}+a_{i} c_{i}\right) \sin \frac{\omega h}{2}+2\left(a_{r} c_{i}-a_{i} c_{r}\right) \cos \frac{\omega h}{2}-\left(a_{r}^{2}+a_{i}^{2}\right) \sin \omega h\right]\right\}
\end{aligned}
$$


and for the refracted wave:

$$
\begin{aligned}
\left\langle\mathscr{P}_{D}\right\rangle= & -\frac{\pi E^{\prime}}{3 H}\left\{8\left(d_{r} e_{r}+d_{i} e_{i}\right) \sin \frac{\Omega H}{2}-8\left(d_{r} e_{i}-d_{i} e_{r}\right) \cos \frac{\Omega H}{2}-\left(d_{r}^{2}+d_{i}^{2}\right) \sin \Omega H\right. \\
& \left.+\frac{m W^{2} H^{2} V^{2}}{10 v_{0}^{2}}\left[2\left(d_{r} e_{r}+d_{i} e_{i}\right) \sin \frac{\Omega H}{2}-2\left(d_{r} e_{i}-d_{i} e_{r}\right) \cos \frac{\Omega H}{2}-\left(d_{r}^{2}+d_{i}^{2}\right) \sin \Omega H\right]\right\}
\end{aligned}
$$

where $a_{r}, b_{r}, c_{r}, d_{r}, e_{r}$ and $a_{i}, b_{i}, c_{i}, d_{i}, e_{i}$ denote the real and imaginary parts of $A, B, C, D$ and $E$, respectively.

\section{NUMERICAL RESULTS}

The deviation of the wave speed in the grid, $v$, from the wave speed, $v_{0}$, in the continuum the grid is supposed to represent is illustrated in Figures 2(a) and 2(b). We see that the higher order three-node elements can represent the wave speed more accurately even at short


Figure 2. Ratio of wave velocities in the grid and the continuum

wavelength $l$. We may also note that the closest representation of the wave speed at short wavelengths is obtained for mass distribution parameter $m=0.5$ for both types of finite elements. These results were obtained from equation (4).

The wave velocity of the refracted wave in the grid, $V$, is plotted in Figures 3(a) and 3(b). We see again considerable differences between the two-node and three-node elements. These results are obtained from equation (6), which can give a solution if $|\cos \Omega H| \leqslant 1$. The end points of the curves in Figures 3(a) and 3(b), correspond to $|\cos \Omega H|=1$. For $H / h$ values beyond the end points there exists no refracted wave, except when $m=1 \cdot 5$. 

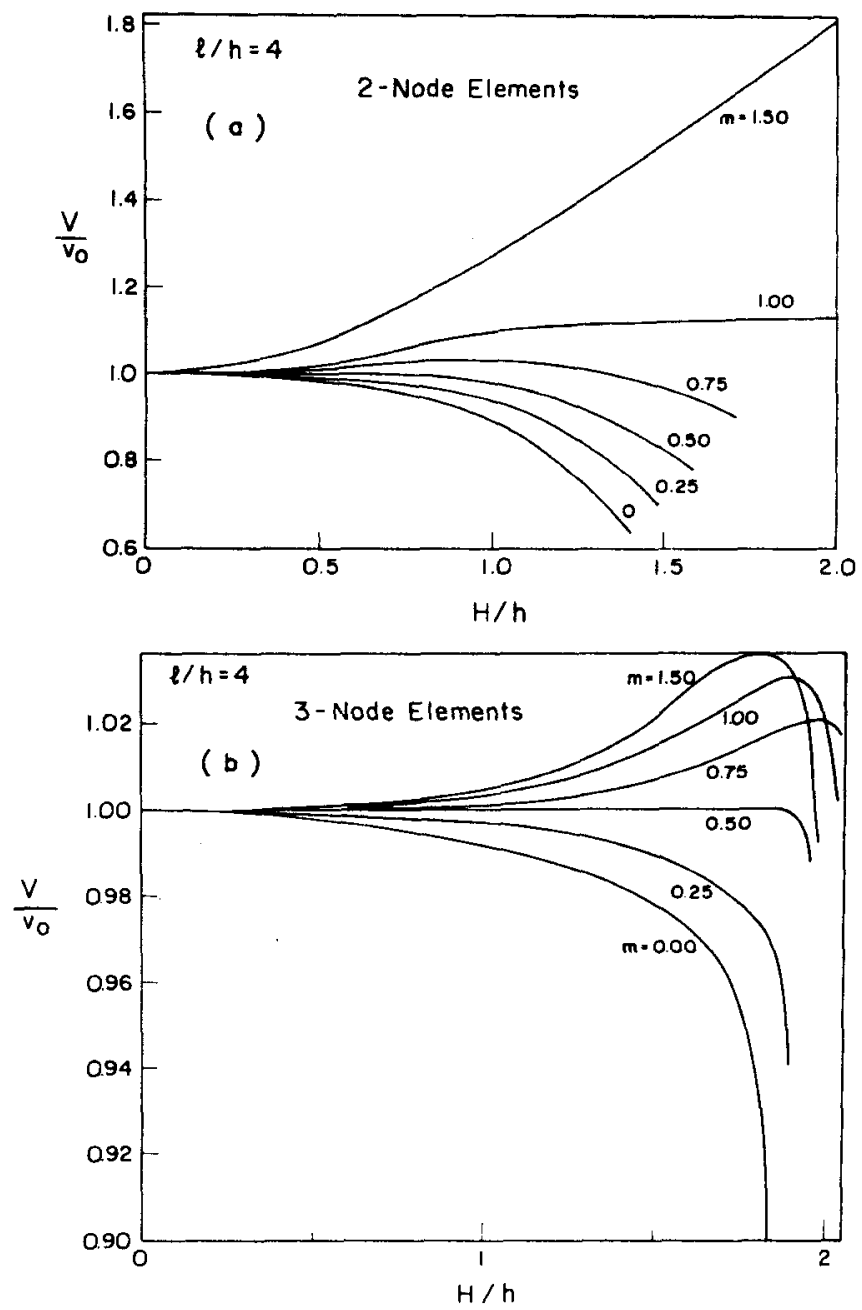

Figure 3. Relative wave velocity of the refracted wave as a function of ratio of element sizes in uniform parts of grid

The amplitudes at the transition points $A_{1}$ and $A_{2}$ and at the end point $D$ are plotted in Figures $4(a)$ and $4(b)$ as a function of the ratio of element sizes of the uniform grids. These figures pertain to $l / h=4$ and are obtained considering two transition nodes. Both the cases when the element size increases and decreases in the direction of the wave propagation are considered. The variation of the element size of the transition elements has been considered as a geometric progression. Figures 5 (a) and 5(b) show similar results for the case when there are five transition nodes between the uniform grid. The values $A_{1}, A_{2}, A_{3}, A_{4}$ and $A_{5}$ denote the amplitudes at the transition nodes, and $D$ the amplitude at the end node. Figures 6(a) and 6(b) show the same results for the higher order elements having three nodes. Two transition nodes are considered here. $A_{1}$ and $A_{2}$ indicate the amplitudes of the boundary nodes of the elements and $B_{0}, B_{1}$ and $B_{2}$ indicate the amplitudes of the interior nodes of the elements. Figures $6(\mathrm{c})$ and $6(\mathrm{~d})$ give the same results for the three-node finite elements and for grids having a transition zone with 10 transition nodes. Again, $A_{2}$ and $A_{4}$ correspond to the boundary nodes of the elements and $B_{0}$ and $B_{5}$ to the interior nodes. 

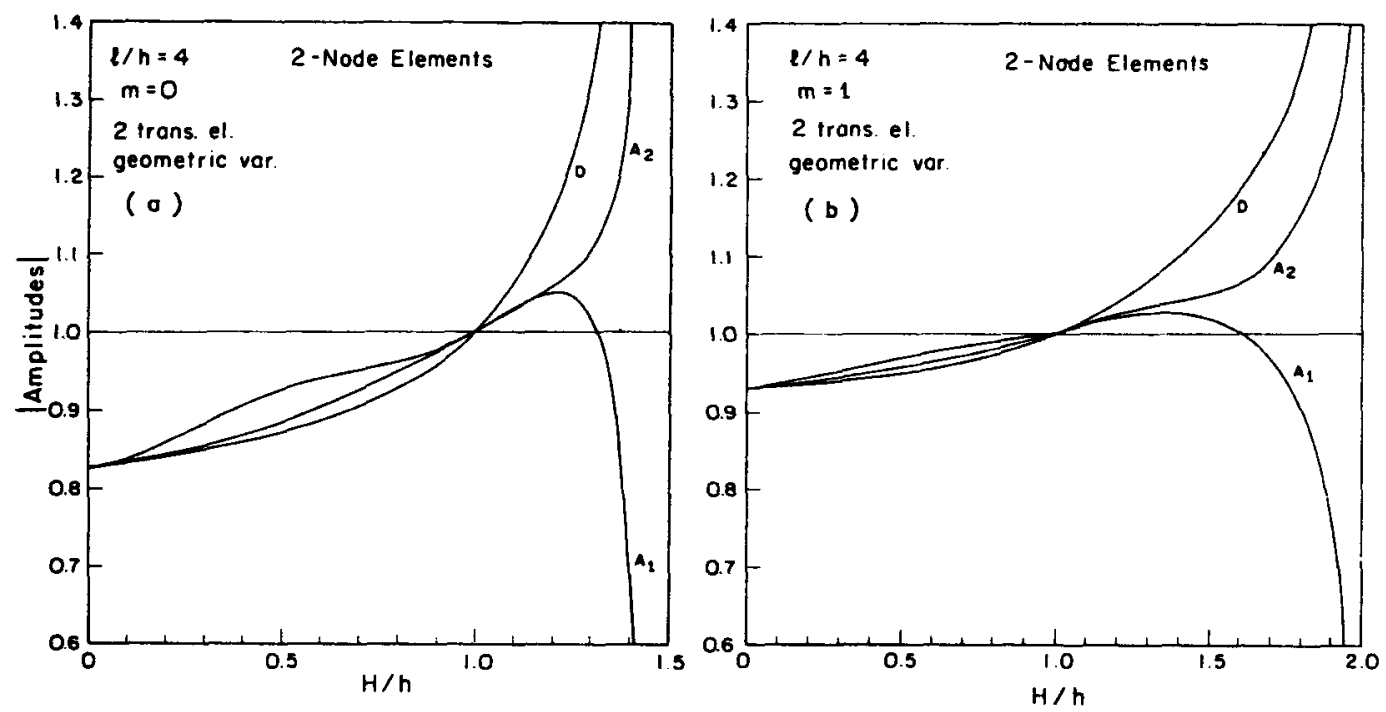

Figure 4. Amplitudes at transition nodes and at end node $D$ for $l / h=4$, two transition points and two-node elements

Of most interest to the computer analyst are the amplitude and the energy flux of the spurious reflected wave which would not occur in a continuum. They are shown in Figures 7-9. In Figure 7, each curve is labelled by a number and a letter; the number indicates the number of the transition nodes between the uniform grids, while the letter $G$ denotes a geometric progression for the variation of element size through the transition zone, and the letter $\boldsymbol{A}$ denotes an arithmetric progression. The curves labelled by 0 refer to the case of no transition zone, i.e. the case of a sudden change of element size from $h$ to $H$, as considered in the previous works. ${ }^{9,10}$ These curves are plotted for a relatively short wavelength, equal to
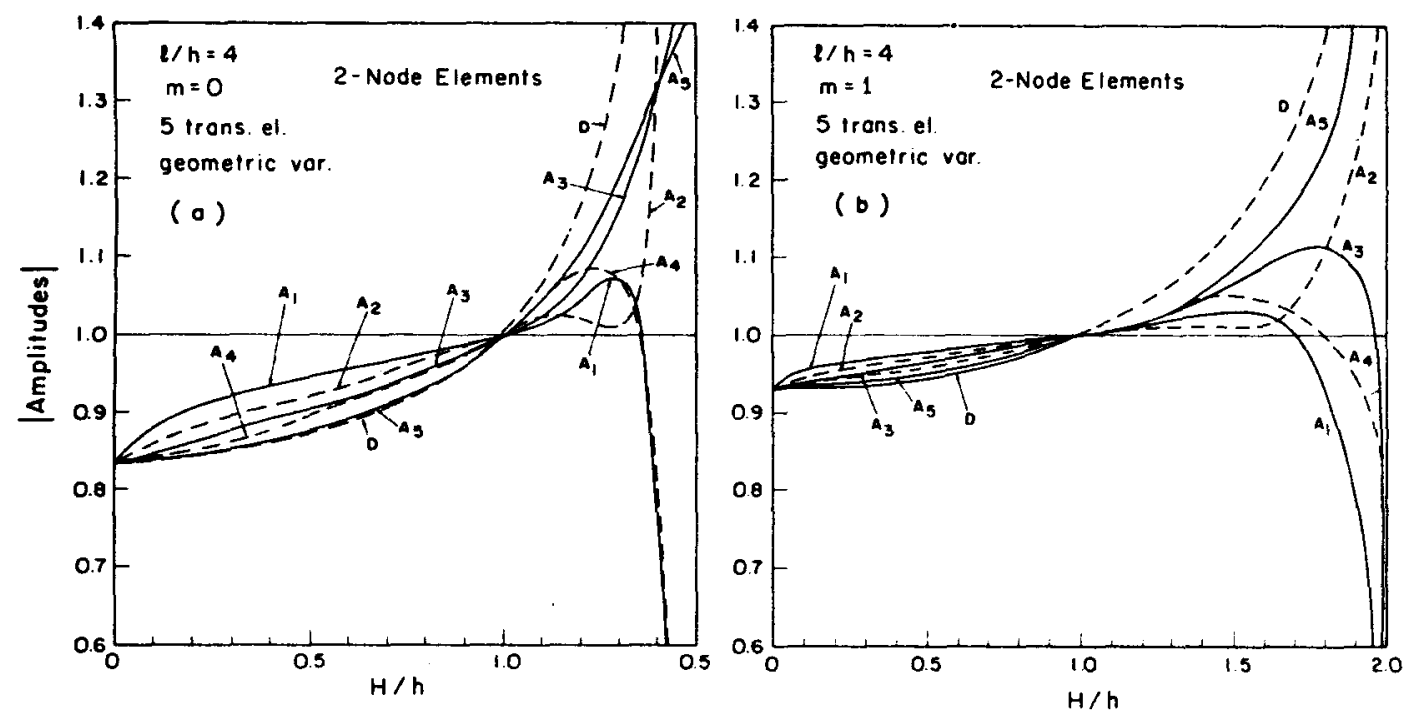

Figure 5. Amplitudes at end node $D$ for $l / h=4$, five transition nodes and two-node elements 

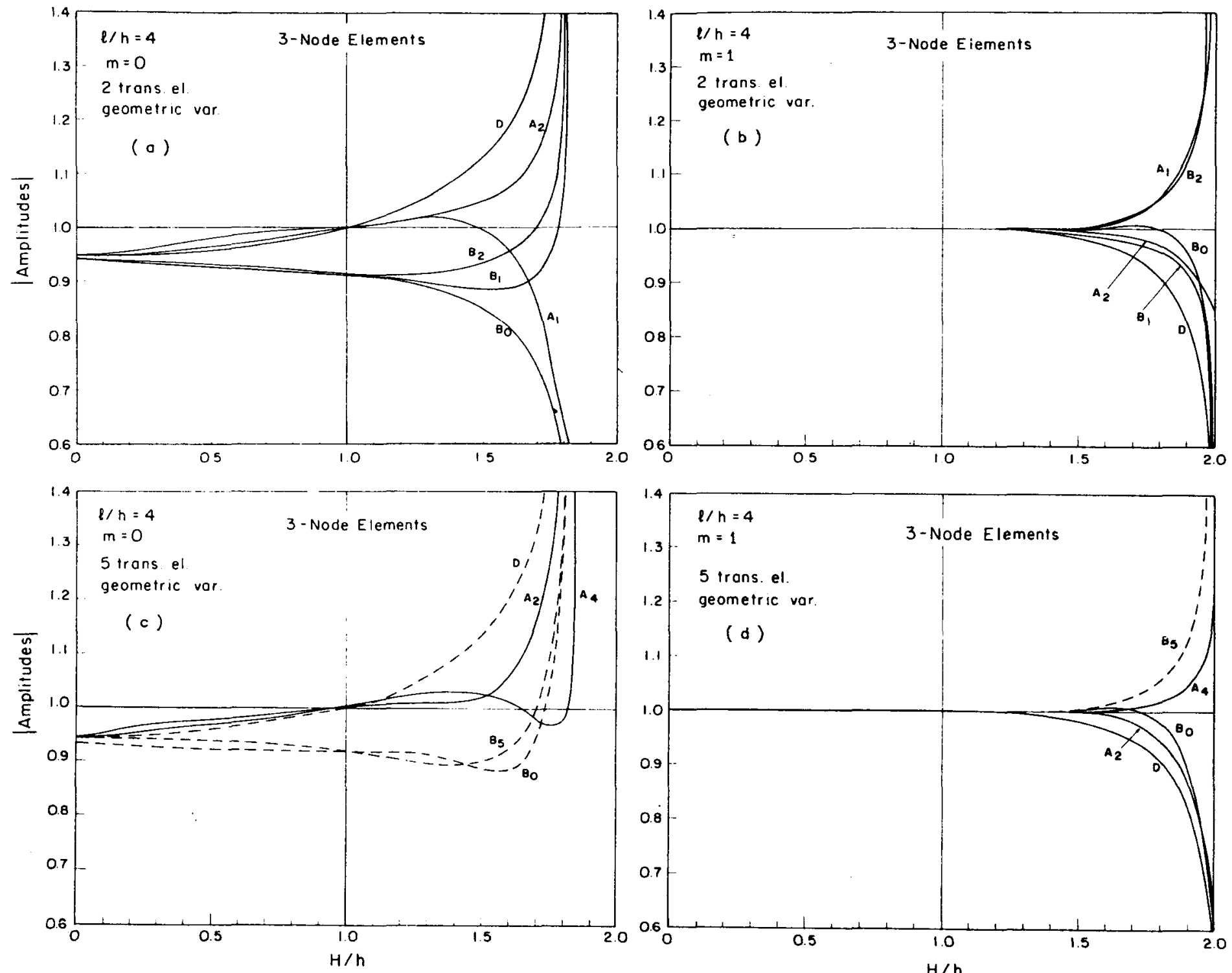

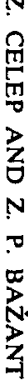

Figure 6. Amplitudes at transition nodes and at end point for $l / h=4$, and three-node elements 

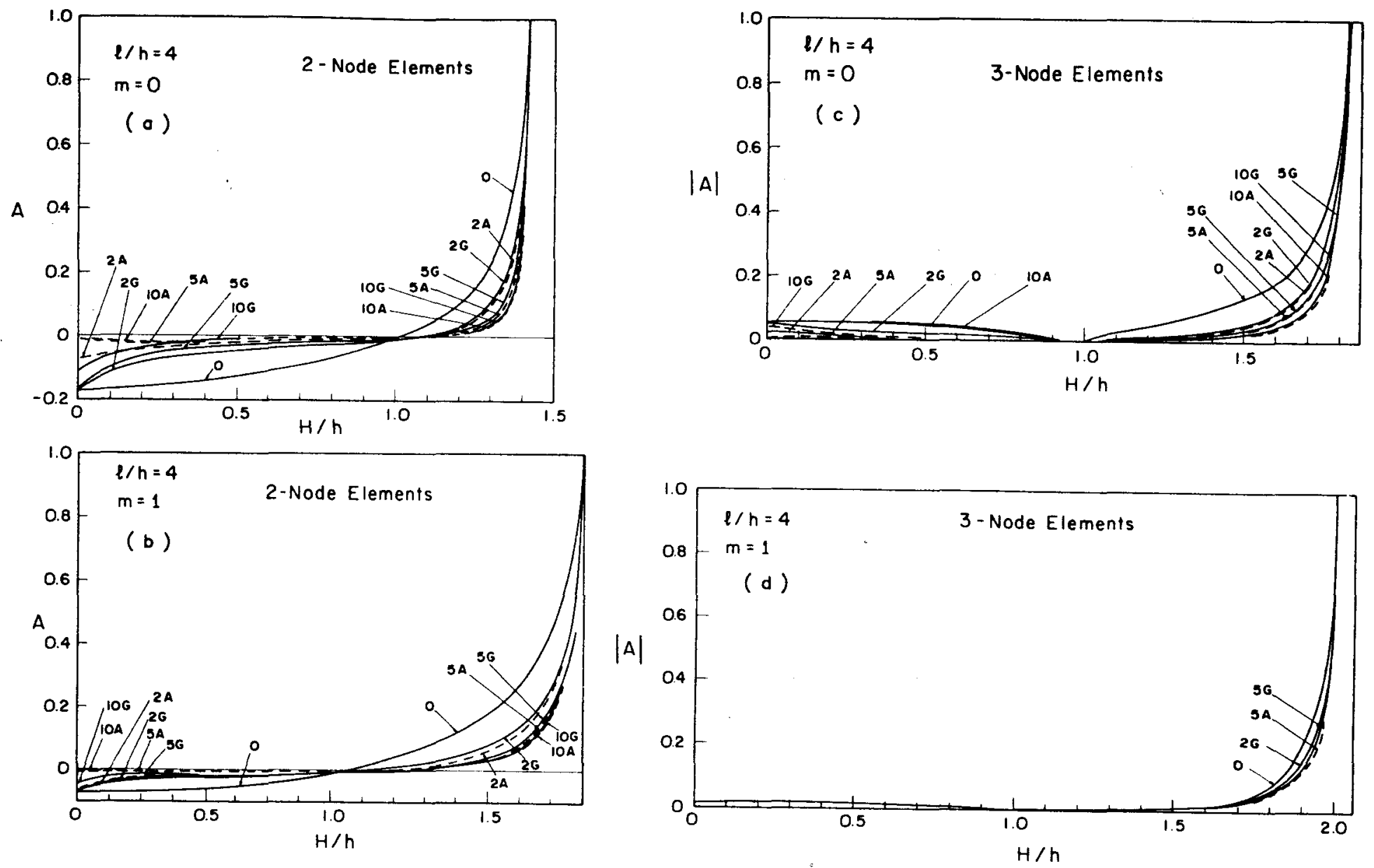

Figure 7. Amplitude of spurious reflected wave in the grid which does not exist in continuum 

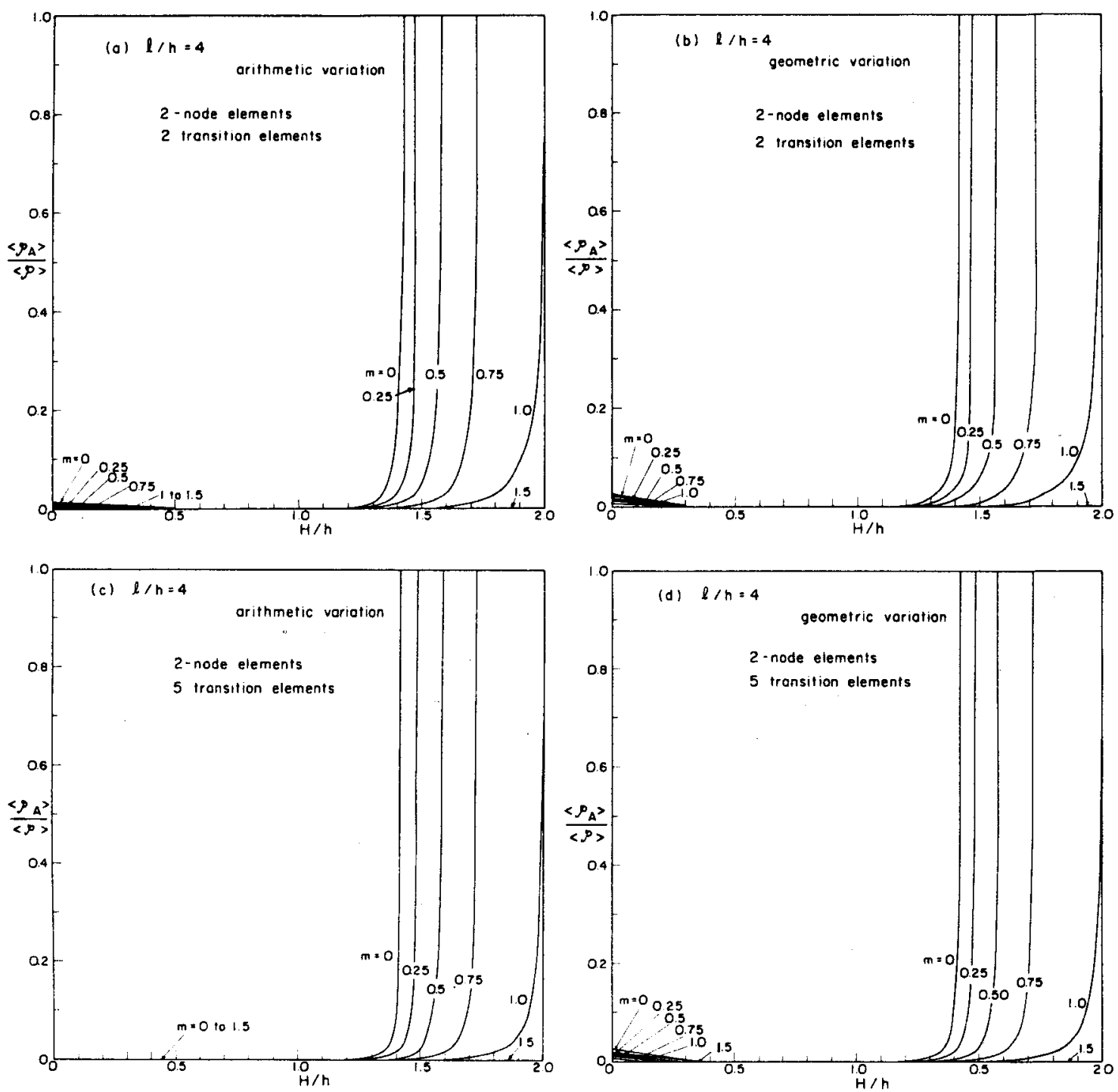

Figure 8. Energy flux of spurious reflected wave for two-node elements

four-times the element size $(l / h=4)$ (the wavelength is defined as $l=2 \pi / \omega)$. For wavelengths longer than $10 \mathrm{H}$, no significant spurious wave reflection occurs, as found before for the case of a sudden change in element size (1).

From Figures 7-9 we see that the amplitudes and the energy fluxes of the spurious reflected waves are rather large. The situation is worse for lumped mass $(m=0)$ than for consistent (distributed) mass $(m=1)$. We also see from these figures that by distributing the change in element size over several transition elements we mitigate the phenomenon of spurious wave reflection, but only to a limited extent. The mitigating effect is pronounced only if the difference in the sizes of the elements in the uniform grid is not too large; otherwise not even a large number of transition elements would help. 

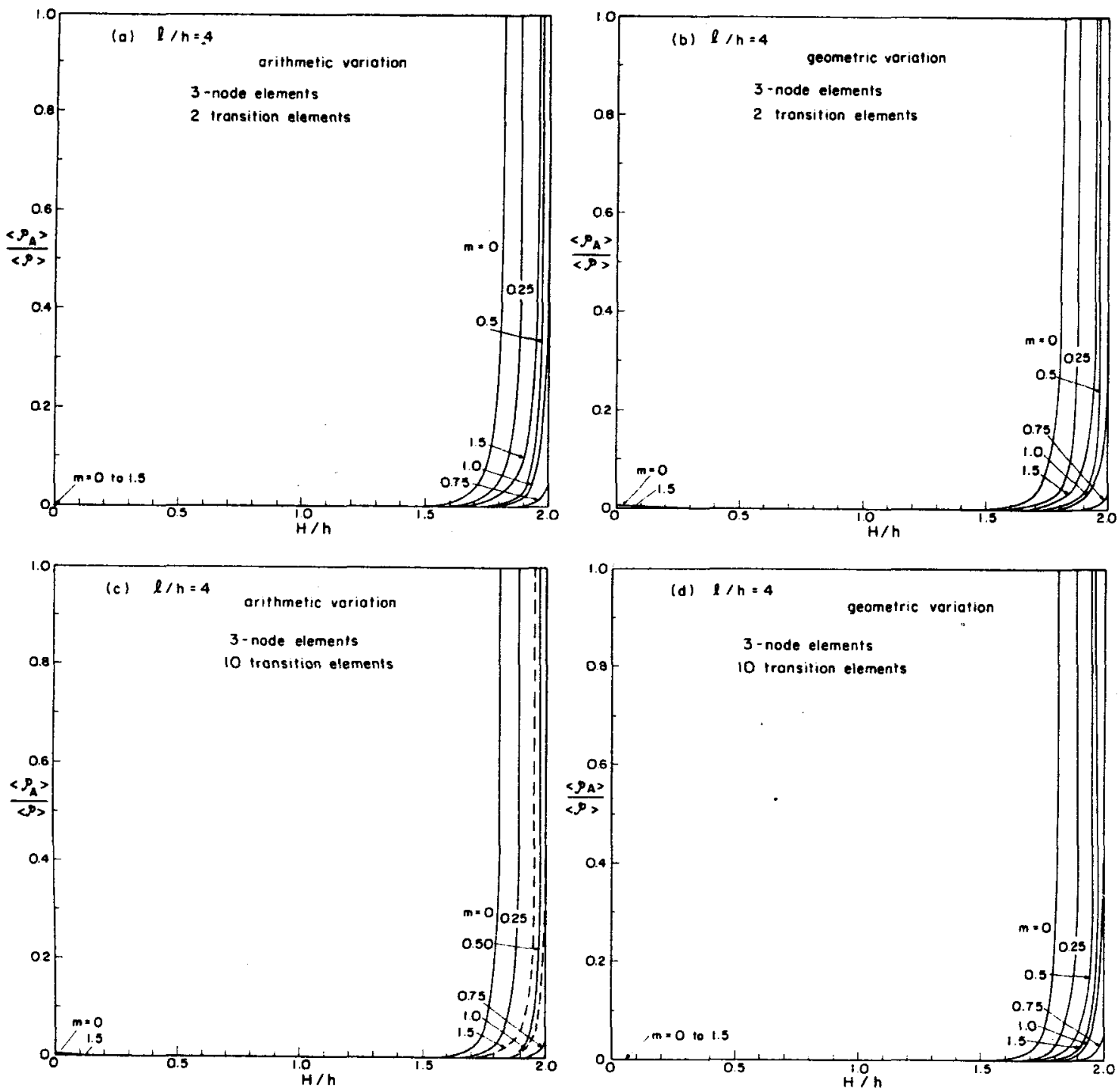

Figure 9. Energy flux of spurious reflected wave for three-node elements

Comparing the two-node and three-node elements, the latter ones are seen to give less spurious wave reflections, but the difference is not very significant. Finally, we may also note that variation of the element size in an arithmetic progression is somewhat more advantageous than in a geometric progression, but the difference is insignificant.

\section{PRINCIPAL CONCLUSIONS}

1. When the wavelength is four times the larger element size, the phenomenon of spurious wave reflection is very significant, while when it is 10-times that size it is unimportant. 
2. Increasing the number of transition elements between the small size and the large size elements of the uniform grids mitigates the phenomenon of spurious wave reflection, but only to a limited extent. The mitigation is significant when the difference between the element sizes in the uniform grids is not very large, but becomes insignificant when the difference exceeds 50 per cent.

3. Varying the element size through the transition region between the uniform grids by an arithmetic progression is somewhat better than by a geometric progression, but the difference is unimportant.

4. For consistent mass the phenomenon of spurious wave reflection is less severe than for lumped mass.

5. The spurious wave reflection is less significant for three-node elements (linear strain) than for two-node elements (constant strain). However, the difference is not large.

6. Finally, in similarity to a previous conclusion, ${ }^{9}$ we may observe that to eliminate the spurious wave reflection the wavelengths which are less than about 10-times the size of the largest element must be filtered out. This can be done in the input, but alternatively this can also be carried out in the output. ${ }^{3}$

\section{ACKNOWLEDGEMENT}

The present problem was solved in connection with fracture mechanics studies sponsored under U.S. National Science Foundation Grant No. CEE-8009050.

\section{REFERENCES}

1. R. D. Krieg and S. W. Key, 'Transient shell response by numerical time integration', Int. J. num. Meth. Engng, 7 (3), 273-286 (1973).

2. T. Belytschko and R. Mullen, On Dispersive Properties of Finite Element Solutions in Modern Problems in Elastic Wave Propagation (Eds. J. Miklowitz, et al.), Wiley, London and New York, 1978, pp. 67-82.

3. N. Holmes and T. Belytschko, 'Postprocessing of finite element transient response calculations by digital filters', Comp. Struct. 6, 211-216 (1976).

4. T. B. Belytschko, 'Transient analysis', in Structural Mechanics Computer Programs, Univ. Press of Virginia, Charlottesville, VA, 1974, pp. 255-276.

5. K. J. Bathe and E. L. Wilson, 'Stability and accuracy analysis of direct integration methods', Int. J. Earthquake Eng. Struct. Dyn., 1, 283-291 (1973).

6. K. J. Bathe and E. L. Wilson, Numerical Methods in Finite Element Analysis, ch. 9, Prentice-Hall, Englewood Cliffs, N.J., 1976.

7. Z. P. Bažant, J. L. Glazik and J. D. Achenbach, 'Finite element analysis of wave diffraction by a crack', J. Eng. Mech. Div. ASCE, 102, 479-496 (1976).

8. R. E. Nickell, 'Direct integration in structural dynamics', J. Eng. Mech. Div. ASCE, 99, 303-317 (1973).

9. Z. P. Bažant, 'Spurious reflection of elastic waves in non-uniform finite element grids', Comp. Meth. Appl. Mech. Eng., 16, 91-100 (1978).

10. Z. P. Bažant and Z. Celep. 'Spurious reflection of elastic waves in nonuniform meshes of constant and linear strain finite elements', Comp. Struct., 15, 451-459(1982).

11. L. Brillouin, Wave Propagation in Periodic Structures, Dover Press, New York, 1946. 